\title{
Why do Psychological Treatments Work?
}

\section{¿Por qué Funcionan los Tratamientos Psicológicos?}

\author{
María Xesús Froján Parga \\ Universidad Autónoma de Madrid
}

Any psychologist, and in particular any clinical psychologist, wants to know why psychological treatments work-in other words, what processes underlie or explain therapeutic change. Answering this question has proved difficult in spite of the many attempts to do so. In this special issue we present a sample of these attempts, formulated by some of the main authors in each area. We may or may not agree with some of these viewpoints, but they are always the result of the sort of thinking or experimenting that must accompany progress in any science.

In the clinical field two main lines of research have been co-existing traditionally. They have conceptualized clinical change in contrastive, and, from our perspective, complementary ways. Result-based research (what works best?) has dominated the field until now and has culminated in the formulation of evidence-based psychological practice; whereas process-based research (how and why do treatments work?), which was forgotten for a while, started to regain strength in the nineties, and has produced the most interesting work so far in this area. It is interesting to know which treatments are better than others, but we submit that it is even more interesting to know what makes the former the best. Knowing why psychological treatments work and what processes explain clinical change will permit a truly sound development of psychotherapeutic intervention.

Research on the therapeutic process leads us to emphasize five main foundational principles of psychological therapy:

1. Reclaiming a fundamentally psychological model of therapy, with functional analysis as a unique, irreplaceable tool. By paying attention to the function of our clients' behavior, we can explain what problems bring them to therapy, and we can design and implement intervention techniques that foster new functions, more adaptive in the long run. Psychologists have at their disposal an analytic and therapeutic strategy which meshes with a unique model and places the therapeutic process in a landscape radically distinct from that of other professions (with which it allegedly competes). Our aim is no longer to push for differences with respect to such and such intervention or to question the organic basis of specific pathologies, but to propose an explanatory and therapeutic model that could cover the totality of human behavior. And here we use the term, "behavior," in its wide encompassing sense, that is, as an interaction between a response (covert or public, cognitive or motor, verbal or emotional) and the context of its occurrence. In this sense, behavior is neither action nor reaction, but interaction.

2. Analyzing verbal behavior during the therapeutic exchange. Although therapy is done mainly through speech, it is only recently that therapists have developed a formal study of the verbal interaction between psychologist and client and a conceptualization of what is said in therapy as clinically relevant behavior (in the sense of the Functional Analytic Therapy presented by Valero, Ferro, Kohlenberg and Tsai, this issue).

3. Considering the clinical situation as a natural context in which problems present themselves as they would outside of the therapeutic session. Not only can we change what people do by changing what they say about what they do; through the actions that they show in therapy, people may display the very same problems that make them look 
for psychological help in the first place. In this sense, the therapeutic situation may constitute a unique context in which contingencies of reinforcement and punishment can be established so as to target problem behaviors.

4. Studying the therapeutic relation as an interactive process which becomes therapeutic in and by itself (in the sense of promoting the learning processes that are ultimately responsible for clinical change). In this issue, Krause, Altimir and Horvath discuss the key concept of alliance; traditionally, this concept (like other equivalent ones) has been analyzed not from a dynamic perspective but by emphasizing characteristics of the client and the therapist that may foster a good therapeutic relation (and yet stand outside of the relation itself). We propose, however, that the development of a positive therapeutic relation or alliance depends on the very interactive process that takes place in the therapeutic sessions. In this sense, this relation or alliance is the result of the therapeutic interaction itself. It is by guaranteeing that this interaction has specific consequences that a good alliance can be obtained. This proposal dissolves the old controversy about which of the therapeutic relation or of the intervention technique has more weight-both are the context though which the learning process occurs.

5. Considering that covert (cognitive) behavior has the same properties as overt behavior and can be studied in the same way. Being covert neither changes the nature of behavior nor makes it unobservable, given that covert behavior can always be observed by one person (ourselves). And covert behavior can always become public, largely but not exclusively through language. Here we will not engage the controversy of whether cognition is identical to language (or whether thinking is silent speaking, to use Marino Pérez's terms in his work, Contingencia y Drama). But we take it to be a fact that in therapy, the main procedure to gain access to clients' cognitions is through what they tell us about what they think. The well known technique of cognitive restructuring, a textbook classic in the "cognitive-behavioral" tradition (with scare quotes to emphasize the redundancy of the formulation) works only through verbalizations that are modified along the course of the Socratic debate, until the conclusion is reached that through changes of verbal behavior the cognitions themselves have changed.

What, then, do psychologists do during therapy? From our perspective, what psychologists do is to engage (or at least try to engage) a sequence of learning processes (Pavlovian as well as operant) that occasion the occurrence in the client of novel behaviors, more adaptive and less problematical. These learning processes involve largely, but not exclusively, the verbal interaction that takes place during the therapeutic session. This conceptualization of clinical intervention as the channeling of learning processes in a therapeutic context has motivated the contextual therapies that have renewed the landscape of behavioral interventions in the last two decades. In one way or another, changes in the client's verbalizations must be used to promote and maintain changes outside of the session, so that the new verbalizations serve as discriminative cues for more adaptive daily behaviors (as Schlinger and Alessi, as well as Salzinger, propose in their contributions to this special issue).

But we can go further in our conceptualization of the therapeutic process and extend it to any type of intervention, regardless of the theoretical model to which it adheres. Learning processes occur, whether we acknowledge them or not, whether we promote them through the application of specific techniques or whether they emerge spontaneously. If the psychologist is aware of these processes, then the probability that they promote change in the desired direction will be higher, and the intervention will be more successful; if the psychologist ignores them, then they will occur anyway, but perhaps at a slower pace or with less guidance from the clinician. Learning processes are to clinical change what electricity is to lightning a house: we can have different types of lamps, switches and bulbs, but eventually what produces light is electricity. Whether illumination is done through a chandelier or a simple bulb is secondary to the circuit that brings electricity to the light source. If we know about electricity, our circuit will be more efficient-otherwise we will keep trying different connections until light eventually goes through.

One last question is worth commenting here: the conceptualization of language as the mechanism 
that allows us to bring past occurrences into the present and thereby give them a new functionality. When analyzing clinical problems, the old distinction between the present and the past loses all meaning; so does any rejection of the behavioral model on the ground that it would neglect historical, and possibly fundamental, aspects of people's problems (namely, their past experiences). From a psychological standpoint, the past and the present are part of a unique, functionally relevant field. When a client speaks of his past experiences (either spontaneously or under the challenge of the therapeutic intervention), his language brings these experiences into the present: they derive their present role through their very inclusion in the present moment (as the client talks, feels, or behaves with a specific morphology). If something bad, however serious or important at the time, happened in the past but is not functionally related to current behavior (pathological or otherwise), then the former cannot explain the latter. Conversely, past occurrences that were previously irrelevant can acquire a central place in our explanation of the current problem, if we can identify their functional role by bringing them into the present through language. A person's learning history is present as soon as it acquires a functional role over current behavior. This functional role can be studied through the verbalizations of the person who describes relations established among various events in her life history. These relations are "present," even though they describe past events.

All of the questions that we have raised in this summary can be found developed at greater length in the articles of the special issue. The authors have emphasized which aspects they consider important in explaining therapeutic change. Caro discusses the clinical process in the light of the model of assimilation of problematical experiences. She concludes that assimilation is related to therapeutic change, whereas lack of assimilation correlates with therapeutic failure. Krause, Altimir and Horvath clarify the concept of therapeutic alliance and evaluate its quality as a function of how clients and therapists attend to different aspects of the relation. Schlinger and Alessi propose to study the changes in client's behavior through the verbal interactions that take place in the clinical context; these authors explain its generalization to the client's daily life in terms of verbal conditioning processes that occur during therapy and can alter the functions of stimuli outside of the clinical session to promote healthier behaviors. In his article, which stresses the importance of operant conditioning, Salzinger defends the use of a single scientific model to explain the effects of any therapeutic intervention. Similarly, Valero, Ferro, Kohlenberg and Tsai emphasize the common roots of third generation therapies, which involve the study of equivalence relations between stimuli, the functional analysis of language, and its influence on cognitive and emotional behavior. Their conceptualization of the therapeutic context as a natural setting that is functionally equivalent to daily life is the basis of Functional Analytic Psychotherapy. Finally, Tonneau questions the foundations of cognitive therapy and proposes an experimental analysis of its concepts. He insists that the operant model is not enough to explain clinical change and reclaims the role of Pavlovian processes.

We would like to thank the authors for their participation in this special issue, which allows us to present a complete panorama of the processes relevant to clinical change, and we hope that it will promote reflection and analysis from all of the actors who work in this area. In spite of the many contrasting differences that can be found among us, we share a common goal: helping our clients to be happier. 